\title{
THE MENACE OF VECTOR-BORNE DISEASES
}

\author{
Retneswari M \\ Department of Social and Preventive Medicine, Faculty of Medicine, University of Malaya, Kuala Lumpur.
}

The crux of the current health problem is the alarming increase of dengue cases in the country. Dengue fever is endemic in Southeast Asia with Malaysia seeing dengue cases surge since 2003. The Health Minister of Malaysia has reported that the acute increase in dengue cases is worrying and could hit the country's productivity, tourism industry and economy.

Despite the concerted efforts, by government agencies and other related organizations, to educate the public on maintaining clean homes and surroundings and conduct spot checks on suspected mosquito breeding areas, Malaysia seems to see a steady rise of dengue cases each year. Lately, the presentation of cases as dengue encephalitis adds to the burden of the problem. Thus, it is just timely that the article on Dengue has been addressed in this issue.

Currently, in the absence of an effective vaccine and drug, reducing the Aedes aegypti mosquito population remains the mainstay for the prevention and control of dengue. Community participation in eliminating breeding sites calls for social marketing in dengue prevention, extensive health education and community outreach. The Malaysian community still appear ignorant towards a clean environment and lack the insight that no amount of government initiatives alone will curb the outbreaks.

The Microbiology Laboratory in the University of Malaya must be commended for advancements in molecular diagnostic tests using two multiplex PCRs allowing detection of the virus from Day 1 of fever. This would allow earlier confirmation of the disease and treatment thus reducing morbidity and mortality.

In view of the unique multiethnic nature of the Malaysian population, the study on genotype variants of the HLA class 1 among the different ethnic groups showing diverse genetic polymorphism would not only reflect the significant role in determining the severity of the infections but also aid to identify ethnically distinct individuals with risk for either disease severity and/protection from severe disease (1).

The presence of four serotypes, problem of immune enhancement and complexity of the disease have hindered the development of a vaccine. The success described in this paper in eliciting human immune response in humanized mice holds promise that developing a vaccine for dengue may not be too far away (1).

All three initiatives namely, government commitment, scientific advancement, and community participation cum empowerment are inseparable in the combat against dengue infections. Malaysians need to understand that newer vector borne diseases like chikungunya have been introduced into the country. This would add to the burden of morbidity and mortality due to vector-borne diseases bearing a direct impact on the health care costs. Combating one disease is bad enough, what more with a new disease to tackle.

Having just dwelled with the alarming increase of dengue cases in the country, it appears that Malaysia being in the Asian region has another burden of reemerging diseases within the country. The paper entitled 'Malaria among Foreign Workers in Selangor (2), Malaysia' clearly indicates the marked increase of malaria cases in the state of Selangor, a rapidly urbanizing state in the country. The fact that $60 \%$ of the malarial cases were contributed by foreign workers, mostly males, active age group as skilled/unskilled workers clearly depicts the apprehension that such an endemic disease could become urban. This may represent only the tip of the iceberg further compounded by the huge number of illegal workers in this country and having skipped medical screening. The high mobility of these workers coming from malaria endemic countries, living or working in densely populated areas and carrying the plasmodium vivax infection which causes relapses reflects the impending health issues in hand. To curb the emergence of

\section{Correspondence :}

Retneswari Masilamani

Associate Professor and Occupational Physician Department of Social and Preventive Medicine Faculty of Medicine, University of Malaya, 50630 Kuala Lumpur Email: retneswari@ummc.edu.my 
such urban outbreaks and keep the disease at bay, the immigration department and the health ministry have to work closely to control the influx of illegal workers and ensure very stringent medical examinations before arrival, followed by intensive medical surveillance by FOMEMA.

The study on work-related disease or disorders noted a prevalence of $57.8 \%$ sleep disturbance among nurses in Melaka General Hospital where shift work was not associated with sleep disturbance as would have been anticipated. Nurses working in intensive unit groups (ICU, CCU, burns unit and high dependency) showed a higher prevalence of sleep disturbance compared to other unit groups which could be explained by their nature of high job demand, maintaining a high state of vigilance, responsibility, stress handling critical conditions and high expectancy while working in these areas (3).

A country is considered to have aged when $7 \%$ of its population is elderly. Malaysian is not far from reaching this status as currently it has an ageing population of $6.3 \%(4)$.

As rightfully addressed by the author on home falls and home environmental hazards among the elderly (4), falls have come to be recognized as a major threat to the safety, health and independence of elderly persons. The prevalence of home falls among the elderly was found to be $25.1 \%$ in this study, posing as a considerable threat to this group (4). Though this study is done in a particular district in Melaka, it should initiate further representative studies to prepare the country towards handling an elderly population who desire good health, independence and an opportunity to lead productive lives, the essential ingredients to a general well being of the elderly.
Apart from the review articles, three case reports on intrahepatic subcapsular haematoma complicating laporoscopic, cholecystectomy (5), intrapulmonary broNchogenic cyst (6) and congenital bilateral aplasia of vas deferns (CBAVD) are presented in this current issue (7).

\section{References}

1. Shymala D. Dengue: Breakbone fever, hemorrhagia or shock. JUMMEC 2008; 11(2): 39-52.

2. Masitah M, Nor Alni MN, Mas Ayu S. Malaria among foreign workers in Selangor, Malaysia. JUMMEC 2008; 11(2): 53-58.

3. Nazatul, SM, Samy I, Moy FM, Nabila AS. Prevalence of sleep disturbance among nurses in a Malaysian government hospital and its association with work characteristics. JUMMEC 2008; 11(2): 66-71.

4. Rizawati M, Mas Ayu S. Home environment and fall at home among the elderly in Masjid Tanah Province. JUMMEC 2008; 11(2): 72-82.

5. Law CW, Ng CLL. Intraheptic subcapsular hematoma complicating laparascopic cholecystectomy. JUMMEC 2008; 11(2): 83-85.

6. How SH, Tee HP, AR Amran, ARM Fauzi. Intrapulmonary Bronchogenic cyst: Single case report. JUMMEC 2008; 11(2): 86-88.

7. Koh PS, Shanggar K, Razack AH, Lee G. Congenital bilateral aplasia of vas deferens (CBAVD): A reminder to clinicians. JUMMEC 2008; 11(2): 89-90. 